\title{
Magnetic Properties of Anion-Radical Salts Based on TCNQ Anion Radical
}

\author{
A. Radváková, M. KajŇAková, A. Feher \\ Centre of Low Temperature Physics of the Faculty of Science UPJŠ \\ and Institute of Experimental Physics, SAS \\ Park Angelinum 9, 04154 Košice, Slovakia \\ D.V. Ziolkovskiy and V.A. Starodub \\ V. Karazin Kharkov National University \\ 4 Svobody Sq., 61077 Kharkov, Ukraine
}

We report the experimental results of anion-radical salts based on TCNQ anion radical. DC magnetic susceptibility measurements in the temperature range from $2 \mathrm{~K}$ to $300 \mathrm{~K}$ and magnetization measurements were performed on two samples: (N-Me-2,5-di-Me-Pz)(TCNQ) $)_{2}$ and (N-Me-2,6-di-Me-Pz)(TCNQ) $)_{2}$ using a Quantum Design MPMS.

PACS numbers: 75.50.Ee, 75.30.Et, 75.40.Cx, 71.70.Gm, 72.80.Le

\section{Introduction}

Organic donor-acceptor radical-ion charge-transfer salts are molecular compounds in which the ionization potential of the donor molecule is relatively small and electron affinity of the acceptor molecule is relatively large. The result is a charge-transfer salt with unpaired electrons appearing on the acceptor or donor, or on both. The existence of unpaired electrons in the lowest unoccupied molecular orbitals (LUMO) allows such a system to be potentially a magnetic insulator or a metal, depending on the crystal structure, the strengths of the various interactions involved, and the temperature.

The most interesting systems studied so far have been based on the acceptor molecule 7,7,8,8-tetracyanoquinodimethane (TCNQ) [1]. Recently, they have been in focus of attention also because of search for genuine organic magnetic semiconductors [2]. Although anion-radical salts (ARS) with TCNQ anions are well known for more than 40 years, they have been designated as quasi-one-dimensional systems up until now. First quasi-two-dimensional salts with pyrazine based cation have been synthesized latterly [3]. Recently, first electric conductivity study of two novel ARS based on TCNQ (N-Me-2,5-di-Me-Pz)(TCNQ) ${ }_{2}(\mathrm{~A})$ and (N-Me-2,6-di-Me-Pz) $(\mathrm{TCNQ})_{2}$ (B) was presented [4]. 


\section{Experimental}

The ARS samples (A) and (B) were investigated in our experiments. Synthesis, crystal structure, IR absorption and electric conductivity studies were reported previously. It was shown that electrical conductivities of these two salts differ by three orders and the conductivity activation energy for both these ARS was estimated and compared with IR absorption spectra [4].

Both of investigated salts have layered structure where cation layers of $[\mathrm{N}-\mathrm{Me}-2,5-\mathrm{di}-\mathrm{Me}-\mathrm{Pz}]^{+}$cation and $[\mathrm{N}-\mathrm{Me}-2,6-\mathrm{di}-\mathrm{Me}-\mathrm{Pz}]^{+}$cation, respectively, alternate along the $c$ axis with layers consisting of TCNQ anion-radicals. The single unpaired electron on $\mathrm{TCNQ}^{-}$occupies the LUMO, which has a $\pi$ level character, and is expected to reside with the same probability on the terminal dicyano methylene carbon groups. Significant difference consists in the organization of anion layers. The planar molecules of anions in (A), parallel to each other, form stacks along the $a$ axis, where they are situated in pairs shifted with respect to each other, and characterized by two overlapping manners. Within the pairs, molecules are eclipsed to a higher degree than between the pairs (Fig. 1a). That is why we can suggest the TCNQ dimerization in stacks, despite the fact that interplanar distances within and between the pairs are almost the same $(3.24 \AA$ and $3.27 \AA$, respectively). Anionic layers in (B) differ from each other by the molecular symmetry therein and the anions overlap in various manners (Fig. 1b).
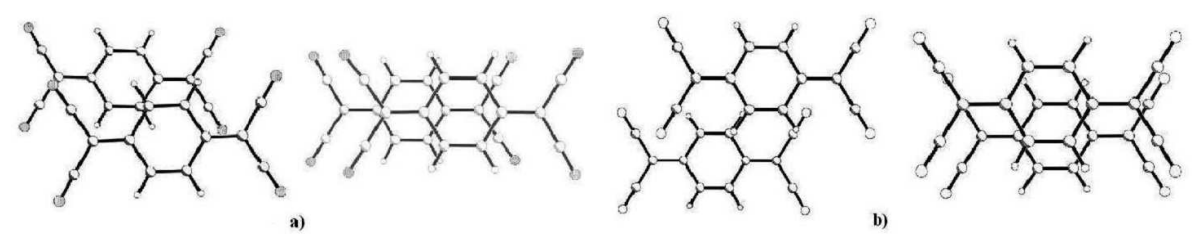

Fig. 1. (a) Overlapping of TCNQ molecules in ARS (A) - between and within pairs. (b) Overlapping of TCNQ molecules in ARS (B) - between and within pairs.

The magnetic susceptibility measurements and magnetization measurements were performed in Quantum Design MPMS. Temperature dependence of susceptibility was investigated in the temperature range from $2 \mathrm{~K}$ to $300 \mathrm{~K}$ in applied magnetic fields $100 \mathrm{mT}$ and $1 \mathrm{~T}$. Diamagnetic contribution to the susceptibility was estimated using Pascal's constants and subtracted from the total susceptibility. Field dependence of magnetization was measured in magnetic field from $0 \mathrm{~T}$ to $5 \mathrm{~T}$ at five different values of temperature.

\section{Results and discussion}

The magnetic susceptibility $\chi_{\mathrm{M}}$ of (A) slowly increases with decreasing temperature and shows a broad maximum at $T_{\max }=38 \mathrm{~K} \pm 1 \mathrm{~K}$, as shown in Fig. 2 . The $\chi_{\mathrm{M}}$ follows the Curie-Weiss law with a Curie constant of $0.568 \mathrm{~K} \mathrm{emu} \mathrm{mol}^{-1}$ 
and a negative Weiss constant $\Theta=-87 \pm 1 \mathrm{~K}$ in the temperature region from $70 \mathrm{~K}$ to $200 \mathrm{~K}$ (inset in Fig. 2). The value of $\chi_{\mathrm{M}} T$ at $300 \mathrm{~K}$ was estimated as $0.484 \mathrm{~K} \mathrm{emu} \mathrm{mol}{ }^{-1}$ and effective moment $\mu_{\mathrm{eff}}=1.98 \mu_{\mathrm{B}}$.

The $\chi_{\mathrm{M}}$ of (B) has a broad maximum at $T_{\max }=35 \mathrm{~K} \pm 1 \mathrm{~K}$ (Fig. 2). $\chi_{\mathrm{M}} T$ vs. $T$ plot (inset in Fig. 2) indicates value of $\chi_{\mathrm{M}} T$ at $300 \mathrm{~K}$ of $0.426 \mathrm{~K} \mathrm{emu} \mathrm{mol}^{-1}$ and $\mu_{\mathrm{eff}}=1.85 \mu_{\mathrm{B}}$. The $\chi_{\mathrm{M}}$ follows the Curie-Weiss law with a Curie constant of $0.56 \mathrm{~K} \mathrm{emu} \mathrm{mol} \mathrm{mol}^{-1}$ and a negative Weiss constant $\Theta=-126 \pm 2 \mathrm{~K}$ in the temperature region from $70 \mathrm{~K}$ to $150 \mathrm{~K}$.

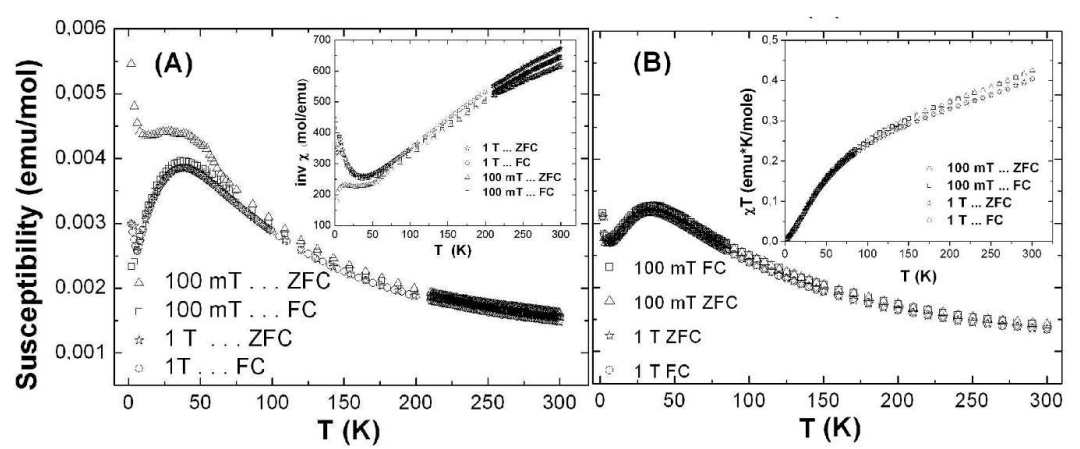

Fig. 2. Magnetic susceptibility of samples (A) and (B) in temperature region from $1.8 \mathrm{~K}$ to $300 \mathrm{~K}$ in magnetic fields $100 \mathrm{mT}$ and $1 \mathrm{~T}$, measured in zero-field cooling (ZFC) and field cooling (FC) regimes. Inset for sample (A) shows plot of inverse $\chi$ vs. $T$ and inset for sample (B) shows plot of $\chi_{\mathrm{M}} T$ vs. $T$.

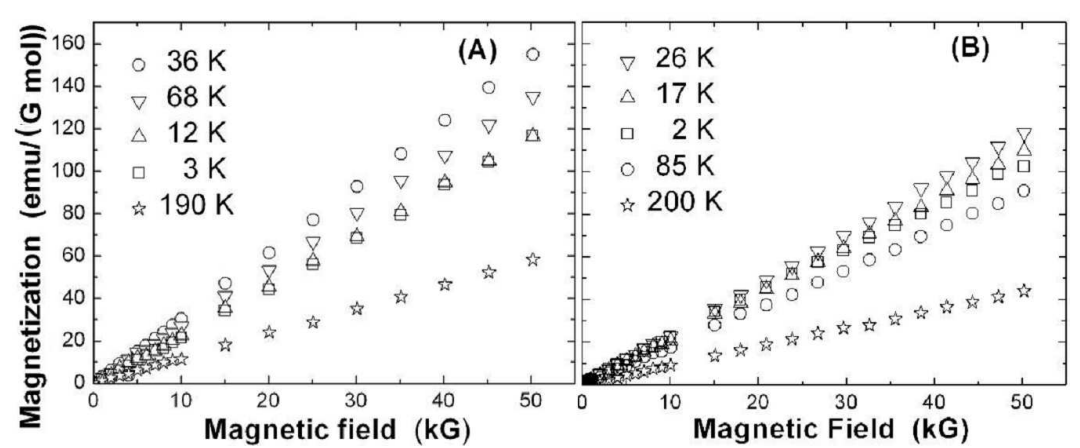

Fig. 3. Field dependence of magnetization at five different temperatures for both samples.

The value of $\mu_{\text {eff }}$ corresponds to one unpaired electron, as expected from the crystal structure. Maximum in susceptibility is probably due to the dimerization of TCNQ anions, whereas the effect is emphasized when magnetic field is applied. Increase in susceptibility below $10 \mathrm{~K}$ for sample $(\mathrm{A})$ and below $6 \mathrm{~K}$ for sample (B) 
is attributed to magnetic impurities, finite chain length or isolated monoradicals, amount of which was estimated as $12.5 \%$ and $5 \%$, respectively. It is unlikely that magnetic impurities could arise during synthesis process.

Field dependence of magnetization is almost linear up to $190 \mathrm{~K}$ (Fig. 3), which means that magnetic interactions persist in higher temperatures. Difference between our two samples is in order of precedence of $M(H)$ dependences at set temperatures.

\section{Conclusion}

Temperature dependences of magnetic susceptibility and field dependences of magnetization were measured on two novel ARS based on TCNQ: (N-Me-2,5-di$-\mathrm{Me}-\mathrm{Pz})(\mathrm{TCNQ})_{2}(\mathrm{~A})$ and $(\mathrm{N}-\mathrm{Me}-2,6-\mathrm{di}-\mathrm{Me}-\mathrm{Pz})(\mathrm{TCNQ})_{2}(\mathrm{~B})$. We conclude that our samples display interesting magnetic behavior: both compounds display weak structural dimerization and show antiferromagnetic type of interaction with unusually large absolute value of the Weiss constant. The strong magnetic correlations are supported by nearly linear behavior of magnetization.

\section{Acknowledgments}

This work was supported by the Slovak Research and Development Support Agency APVV No. 20-005204 and LPP-0102-06. The financial support of U.S.Steel-DZ Energetika Košice is acknowledged.

\section{References}

[1] A.J. Epstein, S. Etemad, A.F. Garito, A.J. Heeger, Phys. Rev. B 5, 952 (1972).

[2] K. Mukai, S. Jinno, Y. Shimobe, N. Azuma, M. Taniguchi, Y. Misaki, K. Tanaka, K. Inoue, Y. Hosokoshi, J. Mater. Chem. 13, 1614 (2003).

[3] D.V. Ziolkovskiy, A.V. Kravchenko, V.A. Starodub, O.N. Kazheva, A.V. Khotkevich, Funct. Mater. 12, 577 (2005).

[4] O.N. Kazheva, D.V. Ziolkovskiy, G.G. Alexandrov, O.A. Dyachenko, V.A. Starodub, A.V. Khotkevich, G.Y. Vasilets, , Funct. Mater. 13, 692 (2006). 